\title{
反铁电/铁电相界附近 $\mathrm{PbLa}(\mathrm{Zr}, \mathrm{Sn}, \mathrm{Ti}) \mathrm{O}_{3}$ 陶瓷性能研究
}

\author{
戴中华 姚 喜 徐卓 冯玉军 王 军 \\ (西安交通大学电子材料与器件研究所, 西安 710049. E-mail: zhdai xjtu@163.com)
}

\begin{abstract}
摘要 采用固相烧结工艺制备了位于反铁电/铁电(AFE/FE)相界附近两个组分的 $\mathrm{PbLa}(\mathrm{Zr}, \mathrm{Sn}, \mathrm{Ti}) \mathrm{O}_{3}$ (PLZST) 陶瓷样品. 研究了电场作用下样品的电致伸缩效应及等静压力对反铁电/铁电相界附近 PLZST 陶瓷相变、介电性能的影响. 在交变电场作用下, 两个样品总应变量分别达到 $0.21 \%$ 及 $0.13 \%$. 随着等 静压力的增加, 反铁电 PLZST 陶瓷铁电/反铁电转变温度降低, 反铁电/顺电转变温度上升; 随着等静压 力的增加, PLZST 铁电陶瓷铁电/顺电转变温度降低.
\end{abstract}

\section{关键词 等静压力 极化 相变 反铁电铁电 $\mathrm{PbLa}(\mathrm{Zr}, \mathrm{Sn}, \mathrm{Ti}) \mathrm{O}_{3}$}

20 世纪中期，Shirane等人 ${ }^{[11}$ 发现PZT二元系固溶 体具有许多优良的特性并进行了深入的研究. Ti的加 入降低了反铁电的相变临界场, 并为室温条件下研 究场诱相变提供了方便. 进一步用 $\mathrm{Sn}$ 替换 $\mathrm{Zr}$ 之后, 由 于 $\mathrm{Sn}$ 的掺入，扩大了四方反铁电相的稳定区域和 $\mathrm{Zr} / \mathrm{Ti}$ 比的调节范围, 有利于反铁电相变特性的研究 和剪裁. Berlincourt在A位掺入微量La对PZST进行改 性得到了PLZST相图(图 1) ${ }^{[2]}$. 之后, 由于铁电、反铁 电材料的应用没有实质性的进展, 再加上研究条件 的限制PLZST基铁电、反铁电陶瓷的研究比较少. 进 入 20 世纪 90 年代, 人们对于PLZST陶瓷材料的研究 逐渐增多. 除了借助现代表征技术对它的结构特征、 相变性能、相变机制等进行深入研究外, 针对其特殊 的相变性能也开发了各种功率器件. 利用其大应变 量的特征可制作 A c t u tors, 如 Rainbow, Moonie ${ }^{[3 \sim 5]}$, 利用其形状记忆特性可制作各种微机械 夹持器 [6,7]. 利用其压制相变过程中电荷释放的突变 特性可制作爆电换能器 ${ }^{[8]}$. 利用其非线性双电滞回线 特性可制作高密度储能器 ${ }^{[9]}$ 等等.

本研究通过对反铁电/铁电相界附近材料组分的 裁剪及调整, 得到具有较大电致应变及较低相变压 力的材料, 研究了等静压力对温度诱导铁电及反铁 电 PLZST 两种材料相变的影响.

\section{1 实验}

选用掺入 $\mathrm{La}$ 离子的锆锡钛酸铅 $\mathrm{Pb}_{0.97} \mathrm{La}_{0.02}$ $\left(\mathrm{Zr}_{1-\mathrm{x}-\mathrm{y}} \mathrm{Sn}_{\mathrm{x}} \mathrm{Ti}_{\mathrm{y}}\right) \mathrm{O}_{3}$ 作为研究体系. 相图选用 Berlincourt 的研究结果(图 1). 在这个固溶体系中存在着四方反 铁电相 $\mathrm{AFE}_{\mathrm{T}}$, 正交反铁电相 $\mathrm{AFE}_{\mathrm{O}}$, 低温三角铁电相 $\mathrm{FE}_{\mathrm{R}}(\mathrm{LT})$ 和高温三角铁电相 $\mathrm{FE}_{\mathrm{R}}(\mathrm{HT})$ 等. 由于相转换

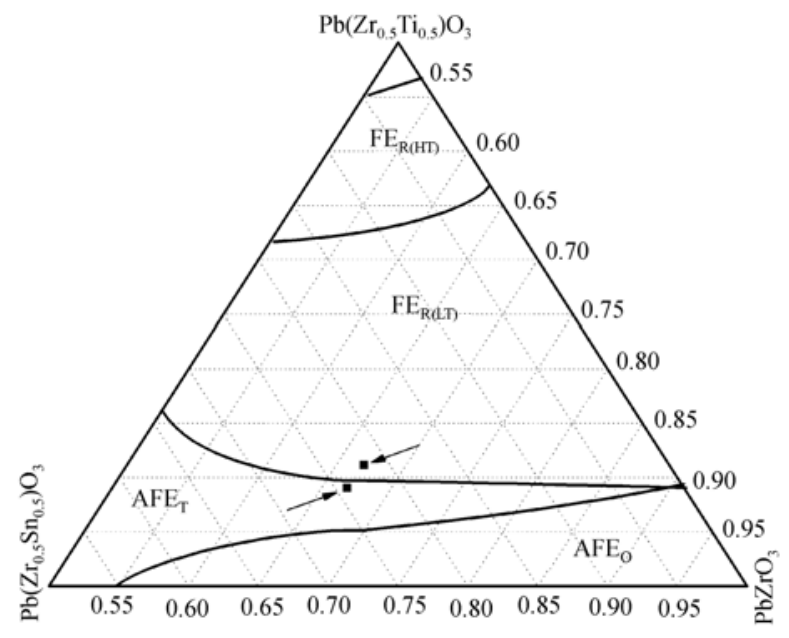

图 1 样品组分在 PLZST 相图中的位置

所需要的外场值很大, 位于正交反铁电相区的材料并 不适合作反铁电/铁电相变研究, 而由于四方反铁电相 组分的陶瓷的能量与低温三方铁电相组分的能量相差 不大, 它们之间的转变在较低的外场下就能够实现.

本研究选用了两个材料配方 $\mathrm{Pb}_{0.97} \mathrm{La}_{0.02}\left(\mathrm{Zr}_{0.67}\right.$ $\mathrm{Sn}_{0.23} \mathrm{Ti}_{0.10}$ ) $\mathrm{O}_{3}$ (A 样品)及 $\mathrm{Pb}_{0.97} \mathrm{La}_{0.02}\left(\mathrm{Zr}_{0.67} \mathrm{Sn}_{0.21} \mathrm{Ti}_{0.12}\right.$ ) $\mathrm{O}_{3}$ (B 样品). 它们的成分位于 PLZST 相图 FE/AFE 相 界附近, 前者位于反铁电一侧, 后者位于铁电区. 样 品采用传统的固相烧结工艺, 经氧化物原料混料、预 烧、成型后, 在 $1240^{\circ} \mathrm{C}$ 富铅气氛中烧结 $2.5 \mathrm{~h}$.

电滞回线的测量采用改进的 Sawyer-Tower 电路, 测试用频率为 $1 \mathrm{~Hz}$ 的正弦波. 高压电场由 Trek Model 609A 产生, 最大输出电压为 $\pm 10 \mathrm{kV}$. 极化样品 的压电系数 $d_{33}$ 用 ZJ-2 型准静态测试仪测量.

在 $2 \mathrm{GPa}$ 等静压装置上测试样品在不同等静压 下介电性能随温度的变化情况, 升温速率为 $3^{\circ} \mathrm{C} / \mathrm{min}$, 
测试频率为 $1 \mathrm{kHz}$. 样品电容 $C$, 损耗角正切 $\tan \delta$ 由 HP4274 测量仪测量.

\section{2 结果与讨论}

\section{1 电滞回线测试}

图 2 是新鲜样品室温下的电滞回线，显示该样品 $\mathrm{A}$ 是反铁电态, 样品 $\mathrm{B}$ 是铁电态. 样品 $\mathrm{A}$ 的 $E_{\mathrm{c}}=0.84$ $\mathrm{kV} / \mathrm{mm}, E_{\mathrm{A}-\mathrm{F}}=1.33 \mathrm{kV} / \mathrm{mm}$, 样品 $\mathrm{B}$ 的 $E_{\mathrm{c}}=0.86$ $\mathrm{kV} / \mathrm{mm}, E_{\mathrm{F}}=0.8 \mathrm{kV} / \mathrm{mm}$. 可见样品 $\mathrm{A}$ 在临界场 1.33 $\mathrm{kV} / \mathrm{mm}$ 下发生了电场诱导的 AFE/FE 转变, 当外电场 去掉后 $(E=0 \mathrm{kV} / \mathrm{mm})$, 试样不能回到反铁电态, 而 是处于诱导的铁电态, 剩余极化强度 $P_{\mathrm{r}}=30$ $\mu \mathrm{C} / \mathrm{cm}^{2}$. 矫顽场较低, 为 $E_{\mathrm{c}}=0.84 \mathrm{kV} / \mathrm{mm}$. 矫顽场较 PZT 和 PZST 陶瓷低, 原因是 $\mathrm{La}^{3+}$ 掺杂形成 $\mathrm{A}$ 位正离 子缺位, 施主杂质 $\mathrm{La}^{3+}$ 成为新的正电荷中心，在其周 围出现一个易受电场激发的导电电子, 使得矫顶场 $E_{\mathrm{c}}$ 降低，同时畴壁容易移动.

将材料制作成厚度 $d=0.5 \mathrm{~mm}$, 直径 $\phi=10 \mathrm{~mm}$ 的样品, 涂上银电极在 $1.2 \mathrm{kV}$ 电压下极化. 由于样品 $\mathrm{B}$ 初始态是铁电态, 内部存在自发极化, 它在较低的

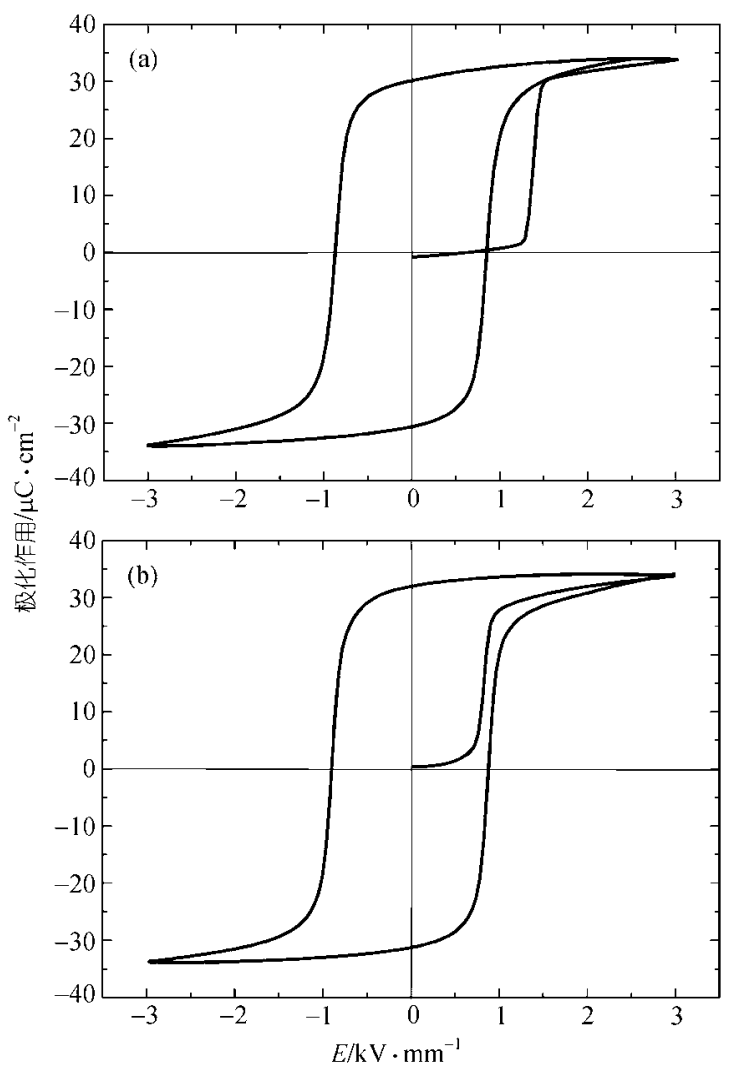

图 2 室温下新鲜样品的第一圈电滞回线 (a) 样品 A; (b) 样品 B
外电场 $E=0.9 \mathrm{kV} / \mathrm{mm}$ 作用下即可以使其内部铁电畴 重新定向.

图 3 是不同温度下测量得到的电滞回线. 在不同 的温度范围内电滞回线表现出不同的特征. 对于极 化状态的 $\mathrm{A}$ 样品, $80^{\circ} \mathrm{C}$ 以下是铁电体, $80^{\circ} \mathrm{C}<T<$ $160^{\circ} \mathrm{C}$ 范围内为反铁电相, 到 $160^{\circ} \mathrm{C}$ 时已经转变为顺 电相. 经过极化的反铁电 $\mathrm{A}$ 样品随温度的升高, 依次 经历铁电/反铁电相变和反铁电/顺电相变. 极化状态 的 $\mathrm{B}$ 样品在 $160^{\circ} \mathrm{C}$ 以下是典型的铁电体的电滞回线, 随着温度的升高在 $160^{\circ} \mathrm{C}$ 附近发生了铁电/顺电相变.

\section{2 电致应变}

电致伸缩效应是高阶机电耦合效应, 比线性的 压电效应微弱. 样品在电压值为 $2 \mathrm{kV} / \mathrm{mm}$ 的交变电 场作用下, 测试频率为 $1 \mathrm{~Hz}$. A 样品最大伸长应变量 为 $0.075 \%$, 最大压缩应变量为 $0.135 \%$; B 样品的最大 伸长应变量为 $0.075 \%$, 压缩应变量为 $0.055 \%$. A 样品 总应变量为 $0.21 \%, \mathrm{~B}$ 样品为 $0.13 \%$. A 样品明显较 B 样品的大, 其中主要是由于 $\mathrm{A}$ 样品的最大压缩应变 远大于 $\mathrm{B}$ 样品的最大压缩应变. 这是由于在交变电 场的作用下 A 样品发生了反铁电态与铁电态之间的
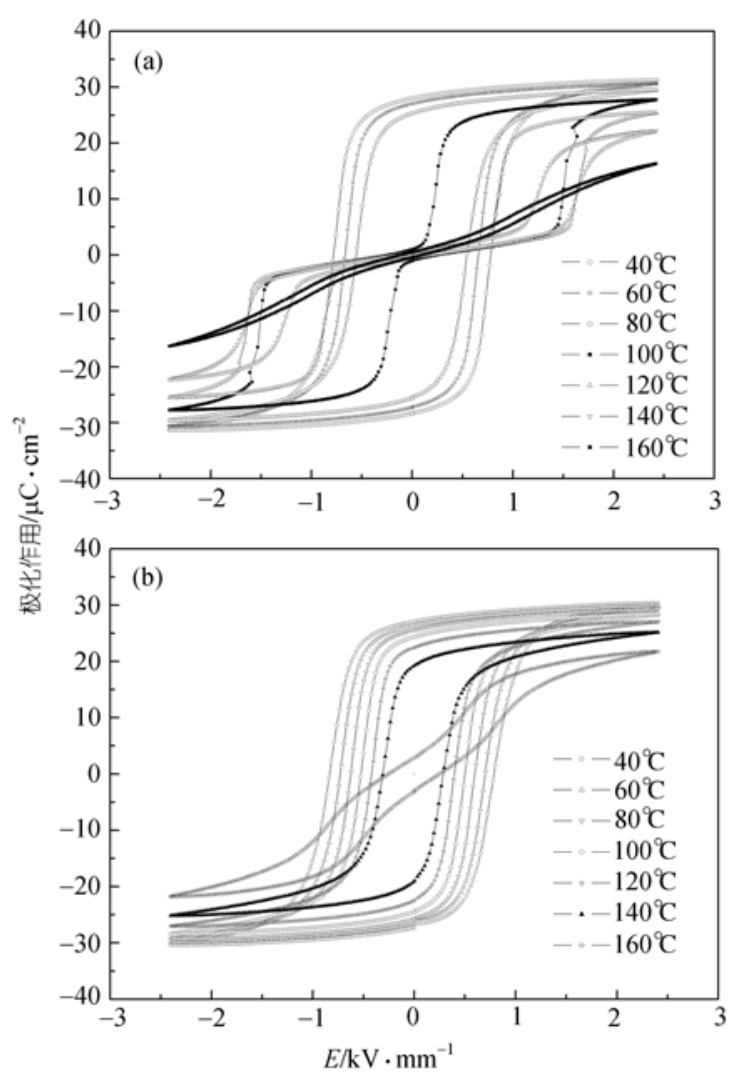

图 3 样品 $\mathrm{A}$ 和 $\mathrm{B}$ 的变温电滞回线 


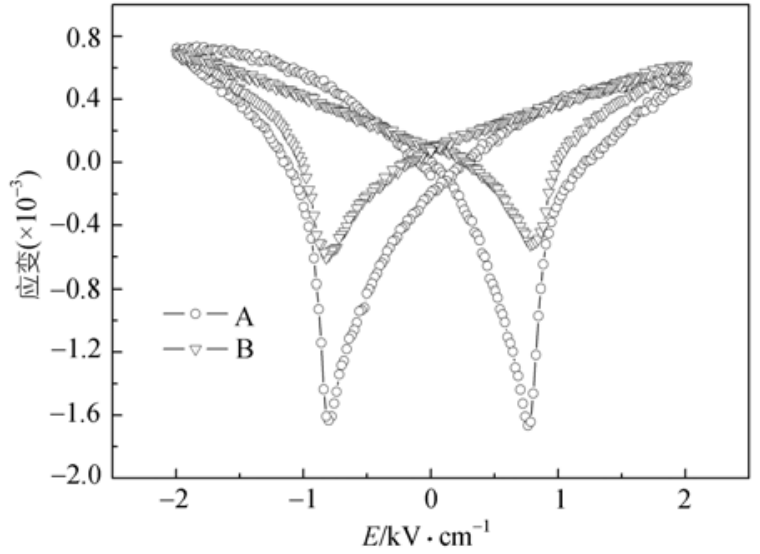

图 4 电致应变曲线

相变, 晶格常数发生变化. PLZST 陶瓷的 $\mathrm{FE}_{\mathrm{R}}, \mathrm{AFE}_{\mathrm{T}}$, $\mathrm{PE}_{\mathrm{C}}$ 三个物相之间的晶体原胞体积关系是 $V_{\mathrm{FE}}>V_{\mathrm{PE}}>$ $V_{\mathrm{AFE}}{ }^{1)}$, 较大的压缩应变是由于 $\mathrm{A}$ 样品在从铁电态向 反铁电态转变过程中原胞体积变小了. 而 $\mathrm{B}$ 样品只 是在电场作用下进行了铁电畴的重新定向.

\section{3 剩余极化与等静压力的关系}

A 样品位于四方反铁电相和三方铁电相相界附 近四方反铁电一侧, 在电场作用下形成了亚稳的三 方铁电相. 测得其压电系数 $d_{33}=95 \mathrm{pC} / \mathrm{N}$. 将极化后 的样品置于压腔中进行等静压实验, 在某个压力值 附近发生压力诱导的相变, 在极短的时间内释放出 电极化时所储存的全部电荷, 通过计算电容上收集 的电荷得到极化样品的剩余极化强度 $P_{\mathrm{r}}$ 值. 图 5 是室 温下样品的剩余极化强度 $P_{\mathrm{r}}$ 和等静压力的关系曲线. 由图可见, 曲线有一个突变, 从相变压力 $150 \mathrm{MPa}$ 开 始释放电荷, 到 $170 \mathrm{MPa}$ 释放电荷结束, 样品的电荷 释放完毕, 重新回到四方反铁电相, 剩余极化强度 $P_{\mathrm{r}}$ 由 $30 \mu \mathrm{C} / \mathrm{cm}^{2}$ 左右迅速下降到约 $0 \mu \mathrm{C} / \mathrm{cm}^{2}$, 具有连续 压致相变的特征. B 样品在 $450 \mathrm{MPa}$ 的等静压力下也 不出现剩余极化强度 $P_{\mathrm{r}}$ 的变化, 这是由于在此压力 下无法实现 $\mathrm{FE} / \mathrm{AFE}$ 的转变, 铁电畴没有重新定向, 因此没有表面束缚电荷释放.

\section{4 等静压力对相变温度的影响}

图 6 为极化样品 $\mathrm{A}$ 在等静压力为 $P=1 \times 10^{5} \mathrm{~Pa}$, 30 和 $60 \mathrm{MPa}$ 时的温谱曲线. 测试频率为 $1 \mathrm{kHz}$. 由图 可知, 介电常数 $\varepsilon_{\mathrm{r}}$ 随等静压力的增加而降低, 在介电 常数峰值 $\varepsilon_{\mathrm{m}}$ 处更加明显. 等静压力增加, 反铁电/顺 电相转变峰更加宽化. 30 和 $60 \mathrm{MPa}$ 时 $\varepsilon_{\mathrm{r}}$ 与温度的关

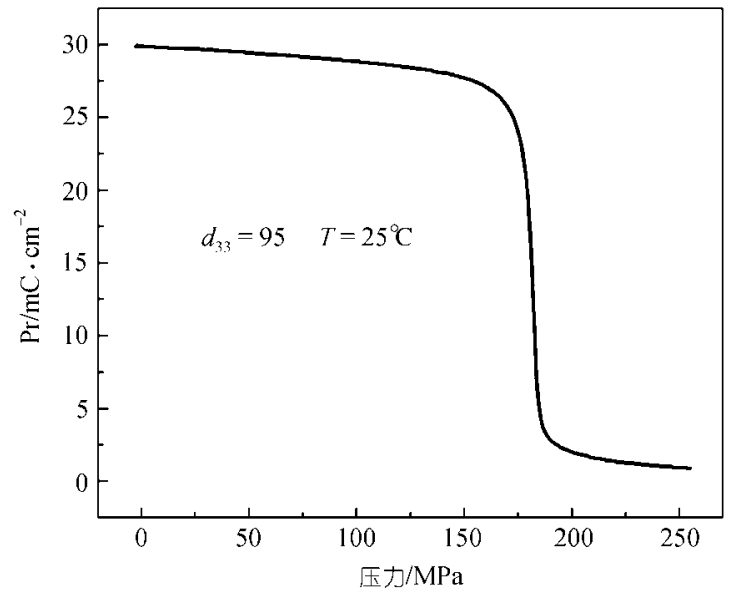

图 5 极化态 $\mathrm{A}$ 样品的剩余极化强度与等静压力的关系

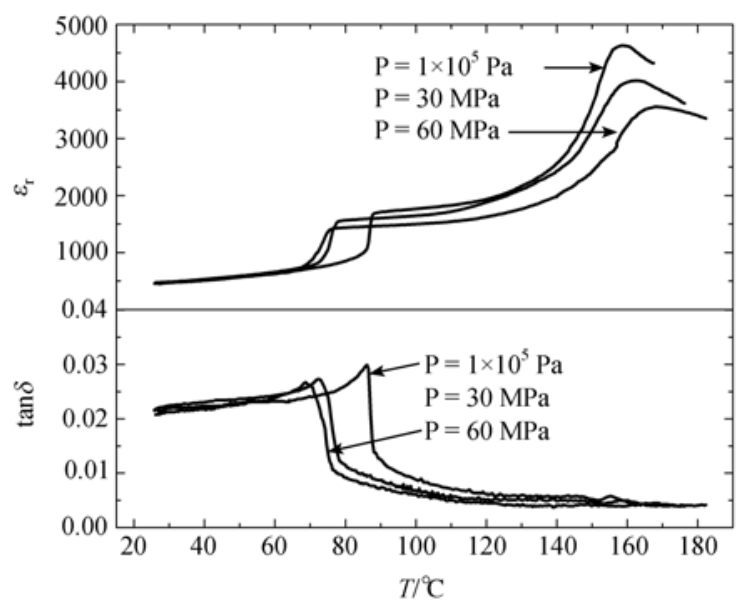

图 6 等静压力对 $\mathrm{A}$ 样品相变温度及介电性能的影响

系不符合居里-外斯定律而满足如下关系:

$$
\varepsilon_{\mathrm{r}}=\frac{k}{\left(T-T_{m}\right)^{\alpha}} .
$$

式中 $k$ 为比例系数, $T_{\mathrm{m}}$ 是 $\varepsilon_{\mathrm{r}}$ 峰值温度, $\alpha>1$, 相变呈 弥散性相变 (DPT) 的特征. Smolensky等人 ${ }^{[10]}$ 认为, $\mathrm{DPT}$ 现象起源于钙钛矿结构 $\mathrm{ABO}_{3}$ 中共同占据B位的两 种离子的无序分布, 结构的无序分布导致晶体内部化 学组分的起伏, 各组分不同的微区具有不同的居里温 度 $T_{\mathrm{c}}$, DPT现象是大量微区 $T_{\mathrm{c}}$ 弥散的结果. 而等静压力 增加了 B位离子的无序分布 ${ }^{[11]}$, 使得PLZST反铁电陶 瓷具有弥散性相变的特征. 随着等静压力的增加, 相 变的弥散性特征更加明显.

等静压力 $P=1 \times 10^{5} \mathrm{~Pa}$ 与 $P=30 \mathrm{MPa}$ 之间的 $\mathrm{FE} /$ $\mathrm{AFE}$ 相变温差 $\Delta T_{\mathrm{FE} / \mathrm{AFE}}=10.5^{\circ} \mathrm{C}$, 而 $P=30 \mathrm{MPa}$ 与 $P=60$ $\mathrm{MPa}$ 之间的 $\mathrm{FE} / \mathrm{AFE}$ 相变温差 $\Delta T_{\mathrm{FE} / \mathrm{AFE}}=2^{\circ} \mathrm{C} ; P=1 \times 10^{5}$

1) 戴中华, 姚喜, 徐卓, 等. 外场下 $\mathrm{PbLa}(\mathrm{Zr}, \mathrm{Sn}, \mathrm{Ti}) \mathrm{O}_{3}$ 陶瓷相变及介电性能行为研究. 稀有金属材料与工程(待发表) 
$\mathrm{Pa}$ 与 $P=30 \mathrm{MPa}$ 之间的 $\mathrm{AFE} / \mathrm{PE}$ 相变温差 $\Delta T_{\mathrm{AFE} / \mathrm{PE}}=4^{\circ} \mathrm{C}$, $P=30 \mathrm{MPa}$ 与 $P=60 \mathrm{MPa}$ 之间的 $\mathrm{AFE} / \mathrm{PE}$ 相变温差 $\Delta T_{\mathrm{AFE} / \mathrm{PE}}=8^{\circ} \mathrm{C}$, 由此总结出等静压力对极化过的 $\mathrm{A}$ 样 品相变的相变温度影响的规律: 随着压力的增大, $\mathrm{FE} /$ $\mathrm{AFE}$ 相变温度向低温方向漂移, 而 $\mathrm{AFE} / \mathrm{PE}$ 相变温度向 高温方向移动. $\mathrm{d} T_{\mathrm{c}} / \mathrm{d} P=(1.33 \sim 2.66){ }^{\circ} \mathrm{C} / 10 \mathrm{MPa}$, 较 $\mathrm{d} T_{\mathrm{c}} / \mathrm{d} P=0.42^{\circ} \mathrm{C} / 10 \mathrm{MPa}$ 的 $\mathrm{PbZrO}_{3}, \mathrm{~d} T_{\mathrm{c}} / \mathrm{d} P=-0.67^{\circ} \mathrm{C} / 10$ $\mathrm{MPa}$ 的 $\mathrm{PbTiO}_{3}$ 对等静压力的灵敏程度更高 ${ }^{[12]}$.

图 7 为 $\mathrm{B}$ 样品在等静压力为 $P=1 \times 10^{5}, 30$ 和 60 $\mathrm{MPa}$ 时的温谱曲线, 样品皆为极化样品, 测试频率为 $1 \mathrm{kHz}$. 由图可知, 峰值介电常数 $\varepsilon_{\mathrm{m}}$ 随等静压力的增加 而下降. 等静压力增加, 铁电/顺电相转变峰宽化. 随 着压力的增大, $\mathrm{FE} / \mathrm{PE}$ 相变温度向低温方向移动. 不同 等静压力下介电损耗 $\tan \delta$ 都有一突降, 对应于 FE/PE 相变.

从压力有利于晶体向体积更小的方向变化的角 度考虑, 而且 PLZST 陶瓷的 $\mathrm{FE}_{\mathrm{R}}, \mathrm{AFE}_{\mathrm{T}}, \mathrm{PE}_{\mathrm{C}}$ 三个物相 之间的晶体原胞体积关系是 $V_{\mathrm{FE}}>V_{\mathrm{PE}}>V_{\mathrm{AFE}}{ }^{1)}$, 等静 压力增大会导致样品铁电/反铁电相变温度降低, 反 铁电/顺电相变的相变温度升高, 同时 $\mathrm{FE} / \mathrm{PE}$ 相变温 度下降. 该实验的结果符合这个规律. 实际上铁电和 反铁电材料两者的 $T_{\mathrm{c}}$ 都可能随压强升高或降低, 这 取决于有序化机构的细节. 铁电与反铁电 PLZST 陶 瓷材料有一强烈依赖于温度的布里渊区中心的光学 模, 该模引起强烈的介电反常, 又有一不稳定的 $q \neq$ 0 的模. 增加压力, 反铁电 PLZST 的 $T_{\mathrm{c}}$ 升高, $T_{0}$ 降低, $T_{\mathrm{c}}$ 处的介电反常显著变小, 即出现高等静压力下的 弥散性相变现象。同时在高压作用下, PLZST 陶

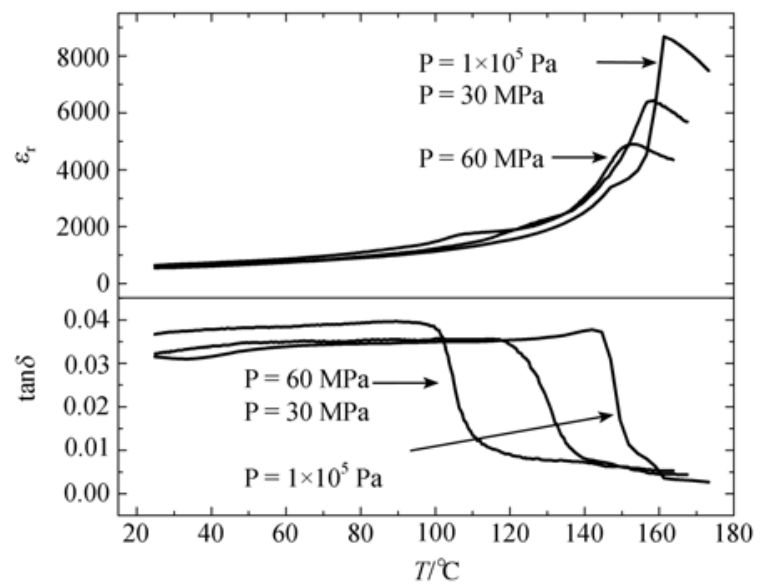

图 7 等静压力对 $\mathrm{B}$ 样品相变温度及介电常数的影响
瓷样品的体积减小, 诱导反铁电/顺电相变的 $q \neq 0$ 的 反铁电模软化, 而同时铁电/顺电相变的铁电模硬化. 从而使得等静压力对铁电、反铁电 PLZST 陶瓷样品 $T_{\mathrm{c}}$ 的影响存在差异.

\section{3 结论}

FE/AFE 相界附近经过极化的反铁电 PLZST 陶 瓷随温度的升高, 由铁电体转变为反铁电体, 最后转 变为顺电体; 极化的铁电 PLZST 陶瓷随温度的升高 由铁电体直接转变为顺电体.

由于反铁电 PLZST 陶瓷在电场的诱导下发生了 $\mathrm{AFE} / \mathrm{FE}$ 相变, 反铁电 PLZST 陶瓷的电致应变较铁电 PLZST 陶瓷大.

随着压力的增大, 极化过的反铁电 PLZST 陶瓷 $\mathrm{FE} / \mathrm{AFE}$ 相变温度向低温方向移动, AFE/PE 相变温度 向高温方向移动; 极化过的铁电 PLZST 陶瓷 FE/PE 相变温度向低温方向移动.

致谢 本工作为国家自然科学基金(批准号: 10474077)和国 家重点基础研究发展规划(批准号: 2002CB613307)资助项目.

\section{参考文献}

1 Shirane G, Suzuki K. Crystal structure of $\mathrm{Pb}(\mathrm{Zr}, \mathrm{Ti}) \mathrm{O}_{3}$. J Phys Soc Jpn, 1952, 8: 7333 7342

2 Berlincourt D. IEEE Trans. Sonics Ultrason. SU-13, 1966: 116

3 Li G, Furman E, Haertling G H. Fabrication and properties of PZST antiferroelectric rainbow actuators. Ferroelectrics, 1996, 188: 233 236

4 Haertling G H. Rainbow ceramics: A new type of ultra-high-displacement actuator. Am Ceram Soc Bull, 1994, 73(1): 93 96

5 Pan W Y, Pan C Q, Zhang Q M, et al. Large displacement transducers based on electric field forced phase transitions in the tetragonal $\mathrm{Pb}_{0.97} \mathrm{La}_{0.02}(\mathrm{Zr}, \mathrm{Sn}, \mathrm{Ti}) \mathrm{O}_{3}$ family of ceramics. J Appl Phys, 1989, 66: 6014 6023[DOI]

6 Furuta A, Oh K Y, Uchino K. Mechanical clamper using shape memory ceramics. Proc.7th ISAF, IEEE, 1990. 525 527

7 Oh K Y, Furuta A, Uchino K. Development and application of shape memory ceramic actuators. Proc 7th ISAF, IEEE, 1990. 528 530

8 Cheng Z M, Lian J Y, Wang Y L. Phase transition studies of PZST ceramics with vertical ferro-antiferroelectric phase boundary. Ferroelectric, 1990, 101: 225 234

9 徐延献. 电子陶瓷材料. 天津: 天津大学出版社, 1993. 25 28

10 Smolensky G A. Physical phenomena in ferroelectrics with diffused phase transition. J Phys Soc Jpn, 1970, 28: 26 37

11 Xu Z, Feng Y J, Zheng S G, et al. Phase transition and dielectric properties of La-doped $\mathrm{Pb}(\mathrm{Zr}, \mathrm{Sn}, \mathrm{Ti}) \mathrm{O}_{3}$ antiferroelectric ceramics under hydrostatic pressure and temperature. J Appl Phys, 2002, 92 (5): 2663 2667[DOI]

12 Chen I W. Structural origin of relaxor ferroelectrics-revisited. J Phys Chem Sol, 2000, 61: 197 208[DOI] 